\title{
Performance investigation of WOFDM for 5G wireless networks
}

\author{
M. F. Ghanim \\ Computer Engineering Department, College of Engineering, University of Mosul, Iraq
}

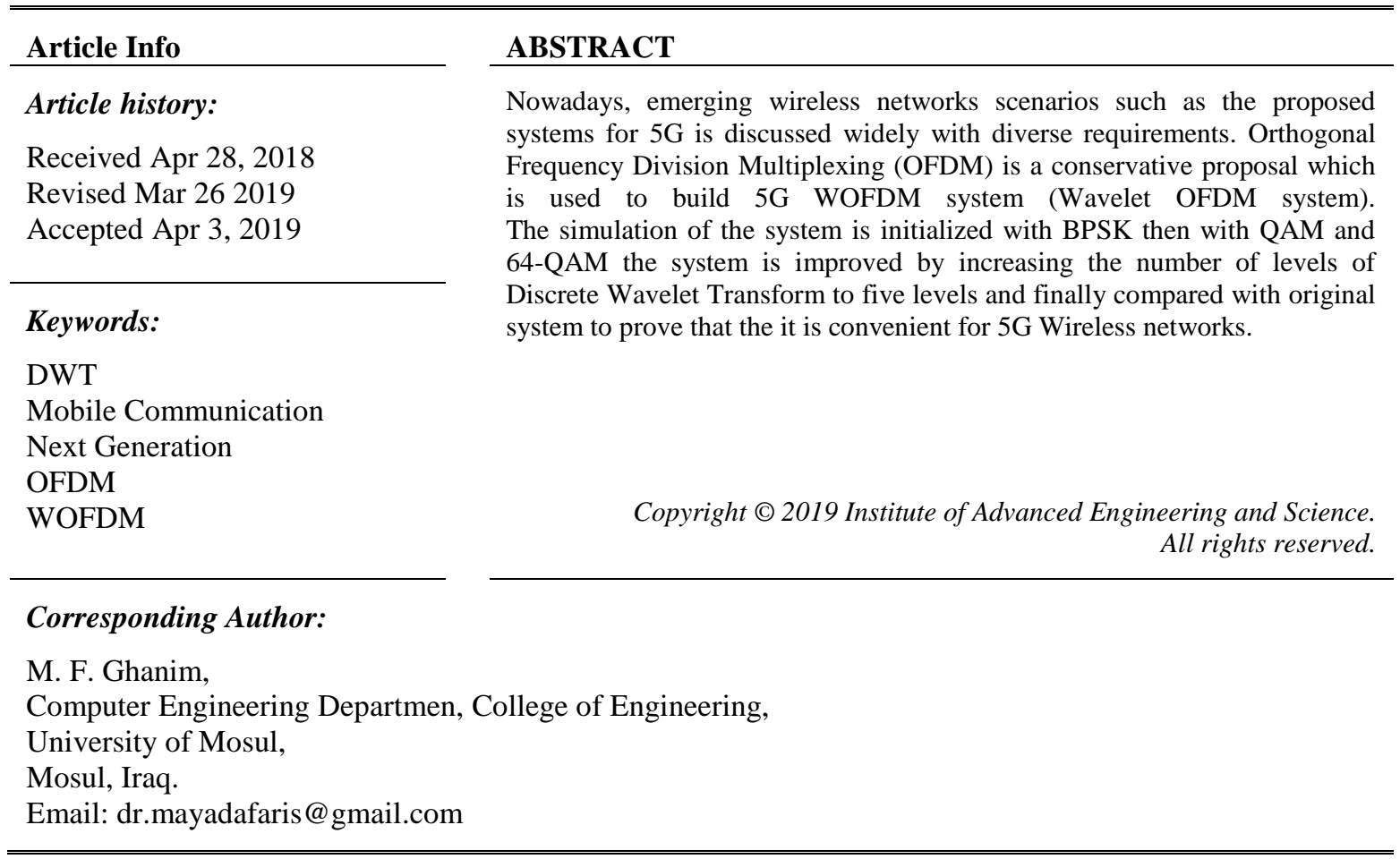

\section{INTRODUCTION}

The concept of modulation and multiplexing system (OFDM) is transmitting data in parallel at the same time through multiple carriers using different frequency bands. These carriers are Fourier complex exponentials in classic OFDM, in other words they are Fast Fourier Transform (FFT) and Inverse Fast Fourier Transform (IFFT), which are used to map symbols to plurality of subcarriers [1]. In fact, any set of orthogonal and normalized functions can be used as carriers of OFDM system such as wavelet. Wavelet OFDM (WOFDM) is designed using discrete wavelet transform (DWT) as carriers. WOFDM transmits symbols in different wavelet spectra. The spectrum is obtained by stretching versions of single spectrum determined by the used wavelet $[2,3]$.

\section{ORTHOGONAL FREQUENCY DIVISION MULTIPLEXING}

OFDM has very large number of subcarrier signals which carry data on parallel data stream. Consequently, each of these subcarriers is modulated with one of the common modulation techniques such as phase shift keying or quadrature amplitude modulation $[4,5]$. The principle of subcarriers (or sub-bands) in OFDM improves the performance of the system. Moreover, the subcarriers enhance the ability to deal with the strict cases of the channel like the attenuation, interference and frequency selective fading in multipath without complex equalization filters $[4,6]$.

The purpose of overlapping of subcarriers in OFDM is to obtain maximum spectral efficiency. Although these subcarriers are orthogonal to one another but overlapping adjacent channels can interfere with one another $[4,7]$. As a result, the subcarriers have the ability to overlap without interfering and accordingly provide the maximum spectral efficiency. Reference [4] proves that the maximum power of each subcarrier is directly proportional to the minimum power of each adjacent channel. The idea of orthogonal subcarriers in OFDM system is clear at the peak of each subcarrier; all the other subcarriers have zero contribution [4]. 


\section{DISCRETE WAVELET TRANSFORM}

Wavelet is widely used for image compression, signal processing, numerical analysis and many other applications such as medical diagnosis and resolution enhancement [8]. Furthermore, wavelet proves that it is a good candidate for $5 \mathrm{G}$ cellular networks if it is combined with OFDM system due to the recent researches in the last few years [9]. The main essence of wavelet transform is decomposing a signal into number of resolutions or sub signals or small wave components called wavelets $[10,11]$. These sub signals are representing the resolutions signal or representing the reminder [12]. Short-time Fourier transform is implemented through employing a sliding fixed size window while, wavelet transform depends a variable window size in analyzing the components of the signal. There is a set of template functions and the signal will be compared with these functions in order to get the similarity. Template functions are generated from the scaling and shift of the base wavelet $\Psi(\mathrm{t})$ as shown in Figure 1 [13].

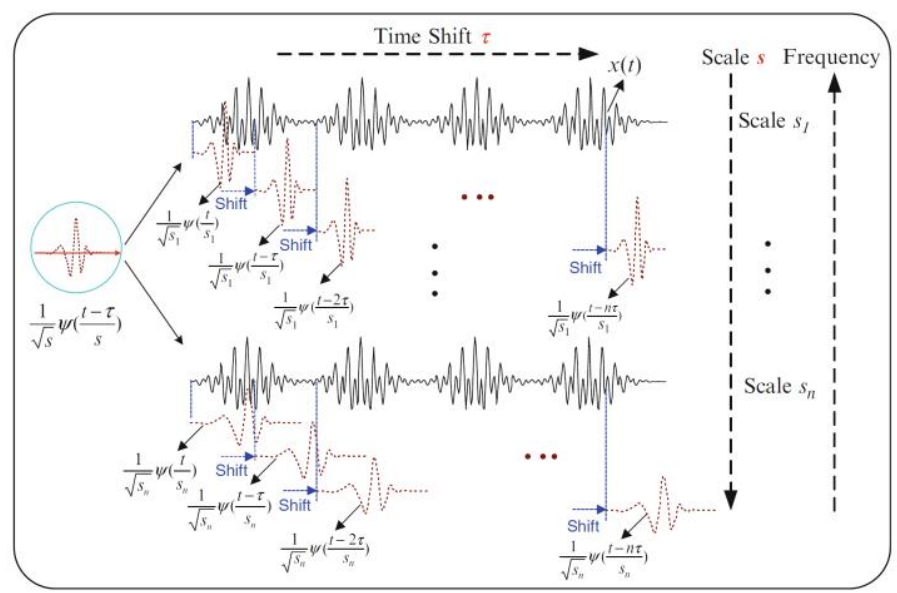

Figure 1. Wavelet transform [13]

Applying inner product, the wavelet transform of $\mathrm{x}(\mathrm{t})$ will be:

$$
w t(s, \tau)=<x, \Psi_{s, \tau}>=\frac{1}{\sqrt{s}} \int_{-\infty}^{\infty} x(t) \Psi^{*}\left(\frac{t-\tau}{s}\right) d t
$$

$\mathrm{S}$ is the scaling parameter and its value must be greater than zero; this parameter is responsible for setting the resolution of time and frequency of $\Psi((\mathrm{t}-\tau) / \mathrm{s})$ which represents the scaled base wavelet [13]. The following points will help in understanding the equation of wavelet transform:

- $\quad s$ is inversely proportional to the frequency.

- $\tau$ is the shifting parameter.

- $\quad \Psi^{*}($.$) is the complex conjugation of \Psi(\mathrm{t})$.

\section{FFT BASED OFDM SYSTEM}

OFDM system usually used in wired and wireless communication systems so it is important to discuss and clarify all the blocks of its transmitter and receiver [14]. The main block in OFDM transmitter is IFFT as shown in Figure 2. Firstly, the input signal will be modulated using any suitable modulation scheme then followed by serial to parallel converter [15]. After that IFFT is applied and the signal s(t) will

$$
s(t)=\sum_{k=0}^{N-1} d_{0, k} e^{j 2 \pi f_{k} t}
$$

Sampling $\mathrm{s}(\mathrm{t})$ at $\mathrm{t}=\mathrm{mTB} / \mathrm{N}[16]$,

$$
\begin{aligned}
& s(t)=\sum_{k=0}^{N-1} d_{0, k} e^{2 \pi f_{k} m T_{B} / N} \\
& y(m)=\left.s(t)\right|_{t=\frac{m T_{B}}{N}}=\sum_{k=0}^{N-1} d_{0, k} e^{\frac{2 \pi k m}{N}}
\end{aligned}
$$




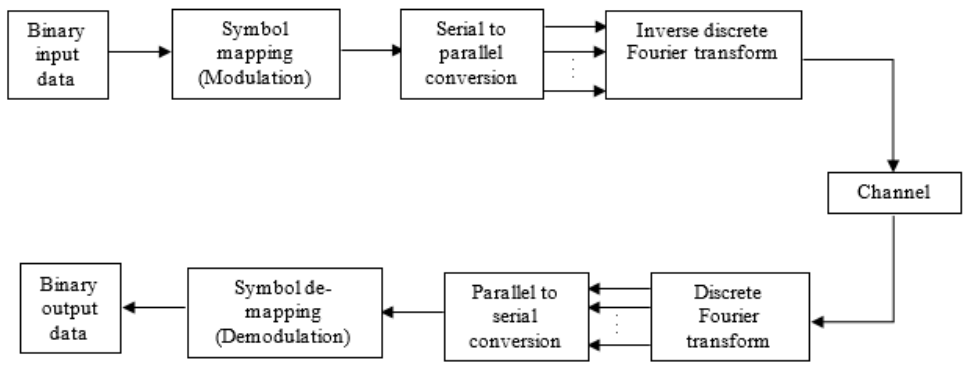

Figure 2. FFT based OFDM system diagram

The sequence $y(m)$ is the IFFT of the data vector di,k. Finally, $y(m)$ is passed to the channel. On the other side, reverse operations are performed at the receiver of OFDM system in order to generate the binary data originally transmitted $\mathrm{d} 0, \mathrm{k}$ as in the (5) [16].

$$
d_{0, k}=\sum_{m=0}^{N-1} y(m) e^{-j 2 \pi k m / N}, k=0,1, \ldots, N-1
$$

\section{WAVELET BASED OFDM SYSTEM}

As mentioned before, FFT based OFDM system divides the bandwidth into subcarriers. These subcarriers are orthogonal and overlapped but in wavelet based OFDM system the principle is different; the data is transmitted depending on multiplexing fundamentals therefore each subcarrier will have its own time and frequency resolution [17] and the system will depend on filter bank instead ofFourier transform to generate the orthogonal subcarriers [18]. The block diagram of wavelet based OFDM system is shown in Figure 3. In the transmitter side, the data is modulated using one of the modulation techniques and converted to parallel in order to apply Inverse discrete wavelet transform instead of IFFT [19]. On the other hand, the received signal from the channel is presented to discrete wavelet transform block then the signal is demodulated to get the original signal with amount of error according to the effect of the noise and attenuation over the channel.

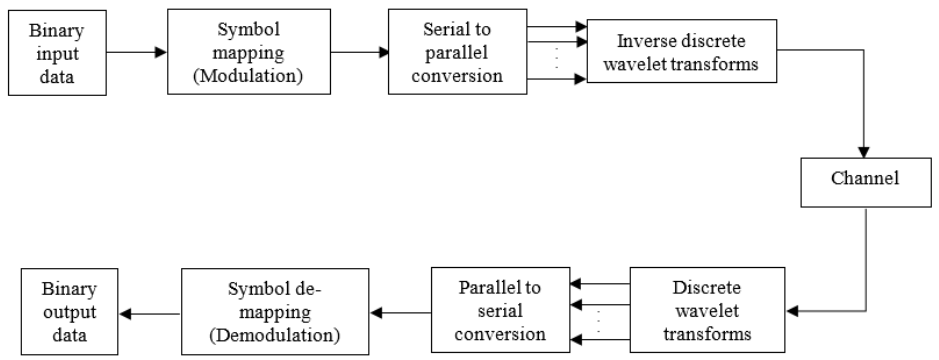

Figure 3. Wavelet-OFDM system diagram

Undoubtedly, the data rate in WOFDM is higher than it in FFT based OFDM [20] because WOFDM is not adding a cyclic prefix (CP) to the symbol before broadcasting over the channel [21] while OFDM employs CP to carry out the channel partition [22]. The system does not require cyclic prefix because of the overlapping subcarriers in time and frequency domain [23]. Also, WOFDM has the advantage of lower PAPR (peak to average power ratio) [24] and lower bit error rate than it in OFDM system [25].

\section{PERFORMANCE ESTIMATION OF WAVELET BASED OFDM SYSTEM}

Before considering the performance of WOFDM system, it is important to illustrate the determinants of the parameters in the simulation. Initially, the selected modulation scheme was BPSK but the results were not convincing enough to adopt the system in this format. Therefore, 64-QAM (i.e. 6 bits per carrier) is used; this scheme of modulation improved the performance of WOFDM system as will be explained later. Moreover, the used wavelet filter is Dmey with BPSK and Haar with $64 \neg$ QAM because this filter is not continuous and not differentiable. In this way, the performance of signals with sudden transitions will be better. The following Table 1 represents the values of simulation parameters. 
Table 1. Simulation parameters

\begin{tabular}{ll}
\hline Parameter & Value \\
\hline Data Length & 256 \\
Number of sub-channels & 256 \\
Modulation & BPSK and 64 QAM \\
Repetition times & 30000 \\
The used wavelet & Haar \\
\hline
\end{tabular}

Figure 4 shows the bit error rate of WOFDM system when BPSK is the modulation scheme the BER is high but the system is stable. At this point, it is better to improve the system and reduce the level of error. For better results, there are two methods to try. The first method is to use Haar filter with 64-QAM modulation scheme, which reduced the error level as shown in the following Figure 5.

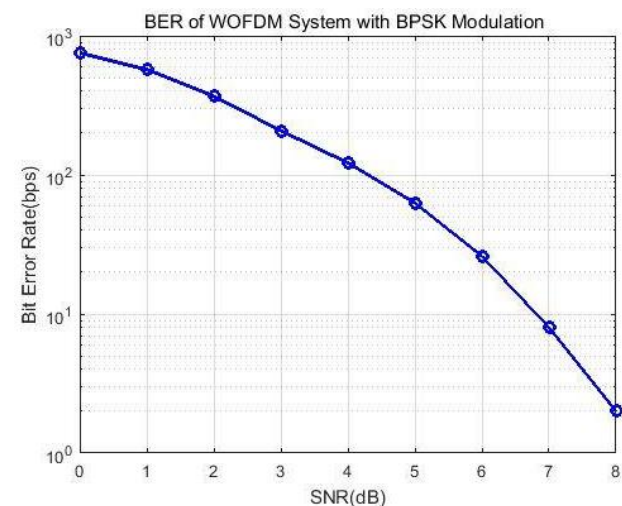

Figure 4. BER of one level WOFDM system with BPSK modulation

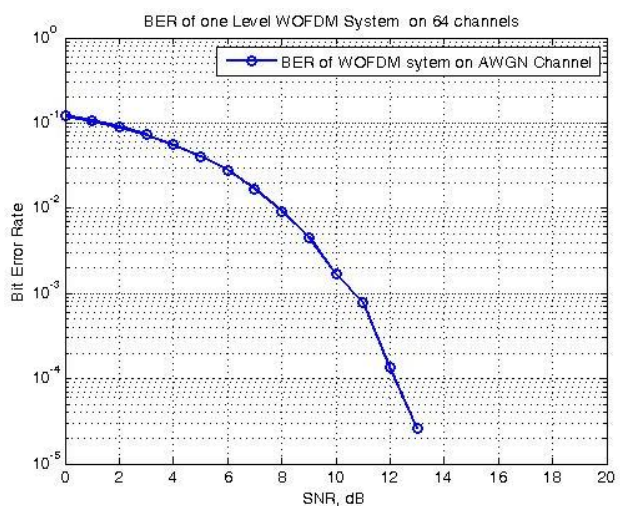

Figure 5. BER of one level WOFDM system with 64-QAM modulation

Evidently, the system is better and still stable with Haar filter and 64-QAM modulation. The second method of improving the system depends on the number of levels of Haar filter in wavelet transform. The number of levels is increased to five instead of one; the system becomes better, stable and BER curve is smooth because the level of error is less when SNR is increasing as shown in the Figure 6. If Figure 6 is compared to Figure 5, the improvement of the performance is clear in Figure 7. BER is reduced and the system is kept stable by increasing the number of DWT levels to five.

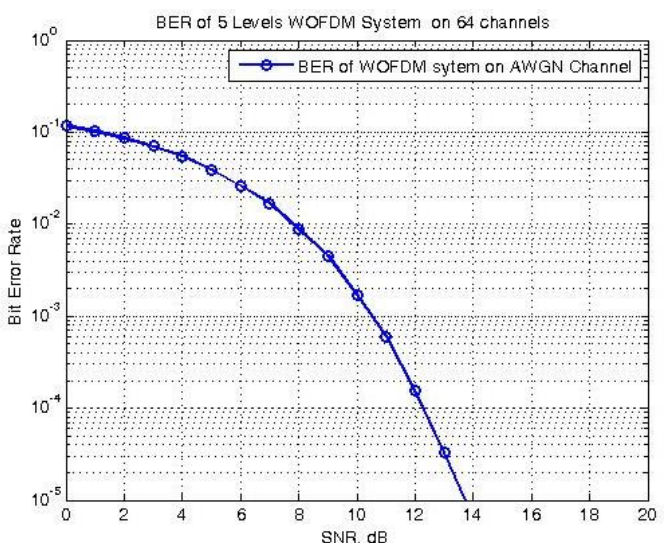

Figure 6. BER of five levels WOFDM system with 64-QAM modulation

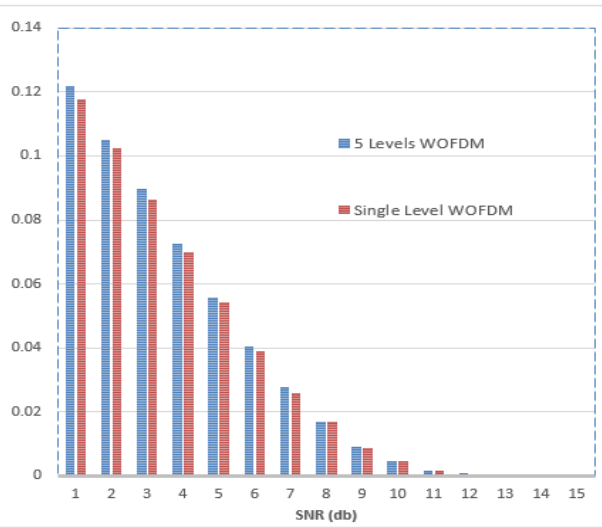

Figure 7. Comparison of one and five levels WOFDM systems in terms of BER 
Finally, Figure 8 illustrates the improvement of the system and reduction of the bit error rate. As a result, this format of WOFDM is good candidate for $5 \mathrm{G}$ networks due to its BER level and stability. The comparison is between the logarithm of BER of WOFDM system with BPSK and BER of WOFDM system with 64-QAM system to show how the BER is dropped to a suitable level for next generation of mobile communications.

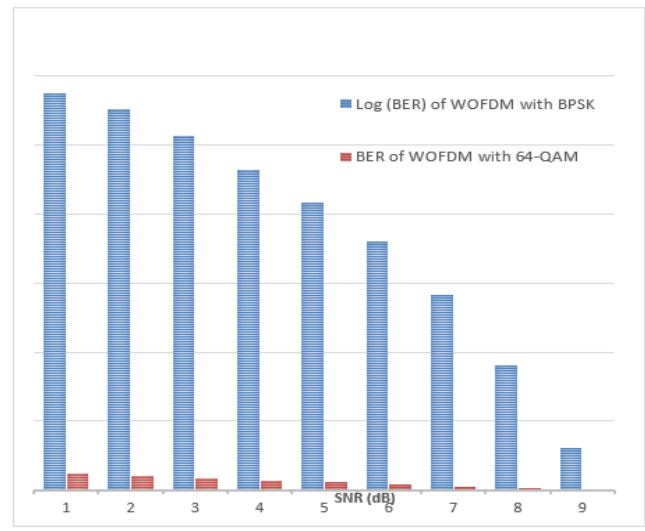

Figure 8. Comparison of WOFDM with BPSK and of WOFDM with 64-QAM in terms of BER

\section{CONCLUSIONS}

In fact, OFDM system is the corner stone of wireless systems in the last several years. The concept of this work is how to improve the performance of OFDM system and reduce BER through using DWT. The system has minimum rate of error when the data sent using five levels of wavelet transform. In addition, it is clear that Haar filter and increasing the number of levels causes the drop of error level. The performance of the system motivates the researchers to work and investigate this method in order to improve other scales such as throughput and PAPR.

\section{ACKNOWLEDGEMENTS}

The author is grateful to University of Mosul, Faculty of Engineering, Departement of Computer Engineering for providing the facilities in carrying out this work.

\section{REFERENCES}

[1] B. Narsimha, K. A. Reddy, "Multi-scale Singular Spectrum Analysis for Channel Estimation of OFDM Transceiver System," IEEE Recent Advances in Intelligent Computational Systems, RAICS 2018, pp. 75-78, 2018.

[2] S. Villalobos, F. Aldana, et al., "A Wavelet-Based OFDM System Implementation on GNURadio Platform vs. an FFT-Based," Springer International Publishing AG, $4^{\text {th }}$ Workshop on Engineering Applications, pp. 201-211, 2017.

[3] S. Shanmugasundaram, "Sub Carrier Analysis for QAM Modulation," Bulletin of Electrical Engineering and Informatics (BEEI), vol. 6, no. 4, pp. 354-357, 2017.

[4] N.S. Sai Srinivas, "OFDM System Implementation, Channel Estimation and Performance Comparison of OFDM Signal," $13^{\text {th }}$ IEEE International Conference on Electromagnetic Interference and Compatibility, INCEMIC 2015, pp. 212-219, 2015.

[5] S. Baig, H. Muhammad, et al., "High Data Rate Discrete Wavelet Transform-based PLC-VLC Design for 5G Communication Systems," IEEE Access, vol. 8, pp. 52490-52499, 2018.

[6] M. Chafii, J. Palicot, et al., "Adaptive Wavelet Packet Modulation," IEEE Transactions On Communications, vol. 66, no. 7, pp. 2947-2957, 2018.

[7] B. Narsimha, K. A. Reddy, "Multi-scale Singular Spectrum Analysis for Channel Estimation of OFDM Transceiver System," IEEE Recent Advances in Intelligent Computational Systems, RAICS 2018, pp. 75-78, 2018.

[8] A. Sharma, T. Gulati, "Change Detection from Remotely Sensed Images Based on Stationary Wavelet Transform," International Journal of Electrical and Computer Engineering (IJECE), vol. 7, no. 6, pp. 3395-3401, 2017.

[9] S. Baig, M. Ahmad, et al., "Dual PHY Layer for Non-Orthogonal Multiple Access Transceiver in 5G Networks," IEEE Access, vol. 6, pp. 3130-3139, 2018.

[10] Y. Medjahdik, Y. Louet, "Impact of selective channels on post-OFDM waveforms for 5G Machine Type Communications," 15 th International Symposium on Wireless Communication Systems, ISWCS 2018, pp.1-5, 2018. 
[11] J. Lee, H. Ryu, "Design and Comparison of Discrete Wavelet Transform Based OFDM (DWT-OFDM) System," Tenth International Conference on Ubiquitous and Future Networks, ICUFN 2018, pp. 881-885, 2018.

[12] J. Lee and H. Ryu, "Performance Comparison between Wavelet-Based OFDM System and iFFT-Based OFDM System," IEEE International conference on Information and Communication Technology Convergence, ICTC 2017, South Korea, pp. 957-960, 2017.

[13] R.X. Gao and R. Yan, "Wavelets: Theory and Applications for Manufacturing," Springer science business media, pp. 26-31, 2011.

[14] P. Manhasa, M. K. Sonib, "Performance of OFDM System under Different Fading Channels and Coding," Bulletin of Electrical Engineering and Informatics (BEEI), vol. 6, no. 1, pp. 54-61, 2017.

[15] Y. Guo, "Wavelet packet transform-based time of arrival estimation method for orthogonal frequency division multiplexing ultra-wideband signal," IET Science, Measurement \& Technology, vol. 9, no. 3,pp. 344-350, 2015.

[16] M. Kavehrad, M. I. Chowdhury, et al., "Short-Range Optical Wireless: Theory and Applications," john wiley, pp.134-135, 2016.

[17] A. Ghaith, R. Hatoum, et al., "Performance Analysis of the Wavelet-OFDM new scheme in AWGN channel," IEEE $3^{\text {rd }}$ International conference on communications and information technology, ICCIT 2013, Lebanon, pp.225-229, 2013.

[18] Y. Ben-Ezra1, D. Brodeski1, "High Spectral Efficiency OFDM Based on Complex Wavelet Packets," $16^{\text {th }}$ International Conference on Transparent Optical Networks, ICTON 2014, pp.1-3, 2014.

[19] M. Chafii, Y. J. Harbi, et al., "Wavelet-OFDM vs. OFDM: Performance comparison," $23^{\text {rd }}$ International Conference on Telecommunications, ICT 2016, pp.1-5, 2016.

[20] O. R. Daoud, "Multiband Orthogonal Wavelet Division Multiplexing: Complexity and Power Peaks Enhancement," IEEE Consumer Electronics Magazine, vol. 8, no. 2, pp.66-70, 2019.

[21] J. Olkkonen, Discrete Wavelet Transforms-Theory and Applications, InTech, Croatia, 2011, pp. 251.

[22] F. A. Pinto-Benel, M. Blanco-Velasco, "Throughput Analysis of Wavelet OFDM in Broadband Power Line Communications," IEEE Access, vol. 6, pp. 16727-16736, 2018.

[23] S. Kar, S. Hussain and X. Fernando, "Wavelet based OFDM for Power line Communication," IEEE 27th Canadian conference on electrical and computer engineering, CCECE 2014, Canada, pp. 1-4, 2014.

[24] O. Daoud, Q. Hamarsheh, "Orthogonal Wavelet Division Multiplexing-Comparison Study," $15^{\text {th }}$ International Multi-Conference on Systems, Signals \& Devices (SSD), pp. 1-5, 2018.

[25] G. G. Rathod, V. S. Ubale, "DWT-IDWT Based Barcode Modulation Method for Verification," $3^{\text {rd }}$ International Conference for Convergence in Technology, I2CT 2018, pp.1-4, 2018. 